\title{
El cómputo cuántico (CQ), la nueva tecnología con las soluciones para el uso masivo de datos, velocidad de cómputo y seguridad informática
}

\section{Quantum computing (QC), the new technology with the solutions for massive data usage, computational speed and computer security}

DOI: $10.46932 /$ sfjdv3n1-043

Received in: Dec 30st, 2021

Accepted in: Jan 1th, 2022

\author{
Dr. José Ruiz Ayala \\ profesor investigador en el Tecnológico Nacional de México \\ Tecnológico de la Laguna en Torreón, Coah. México. \\ E-mail: jjruizad@gmail.com \\ Dr. Luis Héctor García Muñoz \\ profesor investigador en el Tecnológico Nacional de México \\ Tecnológico de la Laguna en Torreón, Coah. México. \\ E-mail: klhgarcía@gmail.com \\ MC Antonio de Santiago Barragán \\ profesor investigador en el Tecnológico Nacional de México \\ Tecnológico de la Laguna en Torreón, Coah. México. \\ E-mail: aga6308@yahoo.com.mx \\ Ing. Alberto Medina Rodríguez \\ estudiante de la maestría en sistemas computacionales en el Tecnológico Nacional de México \\ Tecnológico de la Laguna en Torreón, Coah. México. \\ E-mail: amedinarodriguez@msn.com
}

\begin{abstract}
RESUMEN
Las ciencias que se relacionan con la informática, ya sea en el procesamiento electrónico de datos, o su almacenamiento en grandes volúmenes y la transmisión de esa información por redes que dan la vuelta al mundo casi instantáneamente, se preparan para dar un salto cualitativo y cuantitativo de grandes dimensiones. El desarrollo de sistemas de cómputo basados en la mecánica cuántica así nos lo promete. El computo cuántico será la nueva revolución industrial de este incipiente siglo, algunas grandes compañías se han decantado por esta nueva tecnología que promete solucionar los cuellos de botella que se presentan por el manejo y análisis de este gran volumen de datos (Big Data). Investigaciones realizadas indican que es posible, aunque al parecer los costos de operación y desarrollo indiquen lo contrario. Veamos las características del equipo que se oferta actualmente en el mercado y los avances que se han logrado en este campo, además del lenguaje de programación para simular la programación cuántica; este es el caso del Q\# (Q Sharp de Microsoft). Este lenguaje nos lleva ya a poder enfrentar soluciones complejas intratables en la programación convencional, y nos prepara para la programación de muy alto rendimiento que es la programación cuántica.
\end{abstract}

Palabras clave: Algoritmos Cuánticos, Cómputo Cuántico, Q\# (Q Sharp), Ciencia de los Datos, Big Data. 


\begin{abstract}
The sciences related to computing, whether in the electronic processing of data, or their storage in large volumes and the transmission of this information through networks that travel around the world almost instantaneously, are preparing to make a qualitative and quantitative leap of great dimensions. The development of computing systems based on quantum mechanics promises this. Quantum computing will be the new industrial revolution of this incipient century, some large companies have opted for this new technology that promises to solve the bottlenecks that arise from the handling and analysis of this large volume of data (Big Data). Research indicates that it is possible, although it seems that the operation and development costs indicate otherwise. Let's look at the characteristics of the equipment currently offered in the market and the advances that have been achieved in this field, in addition to the programming language to simulate quantum programming; this is the case of Q\# (Q Sharp from Microsoft). This language leads us to be able to face complex solutions that are intractable in conventional programming, and prepares us for the very high performance programming that is quantum programming.
\end{abstract}

Keywords: Quantum Algorithms, Quantum Computation, Q\# (Q Sharp), Data Science, Big Data.

\title{
1 INTRODUCCIÓN
}

Desde la aparición de la Arquitectura Von Neumann, herencia de este matemático húngaro que logró en 1945 establecer los principios de la computación electrónica, donde una computadora usa largas cadenas de "bits" que codifican ceros o unos, hasta la aparición en estos principios del siglo XXI de la computación cuántica, se han producido una cantidad inmensa de avances en tecnologías, diseños, procesos de fabricación, materiales nuevos, que han llevado a la computación clásica hasta su límite.

Actualmente hay toda una serie de áreas del conocimiento, que destacan por su complejidad y como consecuencia grandes demandas de capacidad de cómputo. En Da Costa, y otros (2020), describen un problema de determinación de aceite en agua, aplicando aprendizaje máquina (Machine Learning), donde la cantidad de cálculos fue resuelta por una supercomputadora. Aquí resalta el hecho de que para un estudio más minucioso, con más variables y variantes del problema, el tiempo de respuesta es crucial (Da Costa, y otros, 2020). Este es un ejemplo de la flexibilidad que representará tener una capacidad de cómputo si bien no ilimitada, mucho mayor a la de una computadora convencional.

Un caso similar lo tenemos en un estudio para resolver de forma radial una ecuación de onda. Además de la recursión, la naturaleza misma del problema es compleja, ya que incluyeron términos homogéneos y no homogéneos de la mecánica cuántica. Para resolverlo utilizaron una supercomputadora y el procesamiento paralelo (Soler-Zapata, Franco-Escudero, \& Arceo-Reyes, 2021). Soluciones a este tipo de problemas, son ejemplos de lo que se espera que el cómputo cuántico nos facilite, tanto por la aplicación de los algoritmos, como el tiempo de respuesta.

La miniaturización de los componentes, incluso la de los procesadores que nos permiten ejecutar todas esas instrucciones de tan elaborados softwares parece o más bien es una realidad que llega a su fin. Luego de varias décadas y muchos avances, la tecnología de la computación entró de lleno en nuestras 
vidas y hoy dependemos para todo, queramos o no, de una computadora incluso de un teléfono inteligente para sobrevivir.

Esto nos ha llevado a manejar grandes volúmenes de información; ante esta situación, el mundo de la computación parece mirar a otros horizontes dónde encontrar soluciones de manejo masivo de datos, velocidad de cómputo y seguridad contra intrusos informáticos. La posible solución se ha encontrado en las leyes de la mecánica cuántica, para poder procesar toda esa información, en lo que ahora se ha llamado computación cuántica, donde las largas cadenas de ceros y de unos se han reemplazado por los bits cuánticos o cúbits. ¿Cuál es la diferencia? Bueno, un cúbit es un sistema cuántico que codifica el cero y el uno en dos estados cuánticos distinguibles. Pero, como los cúbits se comportan cuánticamente, podemos sacar provecho de los fenómenos de "superposición" y "entramado".

La importancia radica en que la cantidad de información contenida en un cúbit, y en particular, la forma en que esta información puede ser manipulada, es fundamental y cualitativamente diferente de un bit clásico. Hay operaciones lógicas que son posibles en un cúbit y no en un bit. El concepto de cúbit es abstracto y no lleva asociado un sistema físico concreto. Pensar en la posibilidad de tener dos estados o dos valores diferentes al mismo tiempo dentro de la mecánica clásica (newtoniana) es imposible. Ya sea una función escalón o una función impulso siempre existirá un intervalo de tiempo entre los cambios de un estado a otro, por lo cual se acepta esta conclusión. Pero dentro de la mecánica cuántica, un sistema cuántico presenta la capacidad para tener múltiples estados en forma simultánea, esto es solo a nivel de partículas subatómicas. A esta capacidad cuántica se le conoce como superposición y será una característica básica de la computación cuántica.

El otro fenómeno cuántico es conocido como “entramado" o Entanglement. Se ha llamado así a una correlación extremadamente fuerte que existe entre partículas cuánticas, tan fuerte, que dos o más partículas cuánticas pueden estar inextricablemente (muy intrincado y confuso) unidas en perfecta armonía, aun si estas se encuentran a grandes distancias entre sí. Bueno las partículas están tan intrínsecamente conectadas que se puede decir que "bailan" en unísono instantáneo y perfecto, aun cuando se colocan en los extremos opuestos del universo.

Y a partir de estos dos fenómenos cuánticos, que serán muy importantes de ahora en adelante, está el futuro de la ciencia de la computación. Su importancia es que serán de gran utilidad para que una computadora cuántica pueda realizar una gran cantidad de cálculos en forma simultánea. La problemática por abordar es el cambio de paradigma de la programación convencional de computadoras vs. la programación de computadoras cuánticas. Para esto, se realizará un análisis exhaustivo del cómputo cuántico para luego evaluar el Quantum Development Kit de Microsoft, Co. De acuerdo con Microsoft (2018), este kit contiene las librerías necesarias para incorporar un simulador de cómputo cuántico en el conocido entorno de desarrollo de Visual Studio 2017. Esto es escribir el código en un lenguaje llamado 
Q Sharp (Q\#, como C Sharp o C\#). Los ejercicios realizados en Q\# serán código para ejecutarse en una computadora cuántica, pero será una simulación porque se ejecutan en un computador común. Microsoft aclara que ya incorpora los algoritmos y estrategias de la versión propia para una computadora cuántica (Microsoft, 2018).

\section{DESARROLLO}

\subsection{LOS INICIOS DEL CAMBIO}

Los esfuerzos de poder realizar cálculos computacionales de una manera cuántica inician en los años 80’s. Entre 1981 y 1982 Richard Feynman proponía el uso de fenómenos cuánticos para realizar cálculos computacionales y exponía que, dada su naturaleza, algunos cálculos de gran complejidad se realizarían más rápidamente en un ordenador cuántico. (Moret, 2005).

La posibilidad de que un computador cuántico pudiese ejecutar algoritmos cuánticos surge a mitad de esa década. Para la siguiente década (90’s) todo esto se hace realidad, se fabrican las primeras máquinas capaces de realizar cálculos utilizando los principios cuánticos y por consecuencia surgen los primeros algoritmos cuánticos y las primeras aplicaciones cuánticas. Fue en 1998 que se presenta una máquina de 2 cúbits o qbits, esto en la Universidad de Berkeley, en California.

Es hasta este siglo que IBM diseña una máquina de 5 qubits donde es posible ejecutar uno de los primeros algoritmos cuánticos diseñados en los 90's. El algoritmo de búsqueda de orden que forma parte del algoritmo de Shor. Con la nueva tecnología este algoritmo se ejecutaba en un solo paso, cuando en la computación tradicional le llevaba muchas iteraciones. El algoritmo de Shor es un algoritmo cuántico para calcular los factores primos de un numero dado. Esto no parecería tener mucha importancia si no es que con la búsqueda de estos factores primos están basados casi todos los sistemas de criptografía que se utilizan en la actualidad.

Los años siguieron pasando y se fueron construyendo computadores de mayor capacidad y se pudieron ejecutar los algoritmos cuánticos diseñados hasta esos momentos que demostraban que este nuevo campo de desarrollo ofrece mayores velocidades de cómputo y por lo tanto parece ser el camino correcto del futuro de las ciencias de la Informática. ¿Pero cuándo estará disponible al usuario común y que costo tendrá?

Finalmente, en 2011, la primera computadora cuántica comercial es vendida por la empresa D-Wave System a Lockheed Martin, por 10 millones de dólares, e IBM anuncia que ha creado un chip lo suficientemente estable como para permitir que la informática cuántica llegue en breve plazo a hogares y empresas. Se estima que en unos 10 ó 12 años se puedan estar comercializando los primeros sistemas cuánticos "asequibles"." (Moret, 2005). Lo que se puede ver hasta este momento es que el desarrollo de máquinas cuánticas va en progreso, pero los costos de venta son muy altos y el desarrollo de algoritmos 
cuánticos es limitado. Los costos asequibles en 10 o 12 años parece ser solo para grandes empresas que tengan la necesidad del manejo de grandes volúmenes de información a grandes velocidades.

No es de sorprender que empresas como Google que es un gigante de la Informática con aproximadamente 3,500 millones de consultas diarias a sus servidores, haya lanzado su microprocesador cuántico llamado Bristlecone, que puede ser el que consiga ese primer hito en la historia de la computación cuántica. Ya lograron avances interesantes con su tecnología de matriz lineal de 9 qubits, con la que trabajaron en una de las limitaciones fundamentales de estos ordenadores: que fallan. ¿Qué fallan? Si fallan, y lo hacen frecuentemente, porque los computadores cuánticos son máquinas extremadamente complejas que son muy sensibles a ruidos y perturbaciones que nos pueden producir errores en los cálculos. Google alcanzo con esa tecnología porcentajes de error de alrededor del $0.6 \%$ con puertos de 2 qbits.

Con este nuevo avance Google esperar alcanzar el liderato en cuanto a computación cuántica pues su nuevo desarrollo posee un microprocesador de 72 qbits, donde espera reducir el porcentaje de error a niveles aceptables para competir con los grandes supercomputadores actuales. ¿Pero qué uso le dará Google a toda esa potencia de computo? Por lo que se sabe hasta hoy, esa potencia está destinada a problemas que por hoy son inalcanzables aun para las supercomputadoras actuales, incluidos aquellos destinados a romper algoritmos de cifrado teóricamente irrompibles.

Otro gigante de las computadoras como lo es IBM, sigue en esta carrera y ha ofrecido que cualquier usuario pueda comprobar el funcionamiento de su procesador cuántico de 5 qbits. Microsoft también participa de esta carrera, pero con un enfoque distinto: desarrollan una computadora cuántica escalable. Para esto utilizan qbits topológicos, basado en aniones.

En concreto son varias las compañías, incluidas IBM, Google, Microsoft, la canadiense D-Wave Systems y la startup Rigetti Computing, con sede en California, que están esforzándose por crear máquinas que las empresas puedan usar. Los problemas de temperatura y de error en los cálculos, son los grandes retos que se tienen para poder hacer esto una realidad que pueda llegar al usuario común.

Algunos expertos en el desarrollo de materiales avanzados como Jonathan Breeze del Imperial College de Londres, dice que los computadores cuánticos deben de reducir ese margen de error que se tiene actualmente en los cálculos para poder usarlos en aplicaciones prácticas. Pero a la vez surge un debate sobre como el aumento de los qbits aumentan el margen de error. Y agrega: si los errores crecen de forma exponencial a medida que se aumentan los qbits entonces esta tecnología está muy lejos de las expectativas que se han generado.

Aún ante estos comentarios de los expertos y la incertidumbre de poder subsanar esos problemas que se presentan en la actualidad, estas compañías siguen inyectando recursos en investigación y desarrollo de los computadores cuánticos de los que se espera sea la nueva gran innovación en materia de 
computación. Y tienen razón para hacerlo, incluso al día de hoy ya se ofrecen algunos productos a la venta en forma comercial, tal es el caso de D-Wave System que ofrece su modelo D-2000Q.

Pero vamos a ver qué es lo que esta compañía (D-WAVE, 2018) ofrece en el mercado y en que ramas de la ciencia proponen su aplicación. La siguiente información es tomada de los manuales de la compañía donde oferta sus productos ya que resulta imposible tener acceso a uno real por parte de un usuario común. D-Wave System es una compañía canadiense pionera en el desarrollo de computadores cuánticos que se autodenomina líder en el desarrollo de sistemas de computadores cuánticos, así como de software, es la única compañía en el mundo que ofrece productos de manera comercial. Y agregan que ellos están ciertos que la computación cuántica permitirá soluciones a los más desafiantes problemas en el campo científico, comercial y de defensa nacional.

Además, los sistemas de D-Wave están siendo utilizados por algunas de las organizaciones más avanzadas del mundo, incluyendo Lockheed Martin, Google, NASA Ames, USRA, la Universidad del Sur de California y Los Álamos Laboratorio Nacional. D-Wave tiene actualmente más de 140 patentes otorgadas en los EE. UU. Y ha publicado más de 90 artículos científicos, muchos de los cuales han aparecido en las principales revistas científicas.

\subsection{COMO TRABAJA}

Los sistemas D-Wave utilizan un proceso llamado quantum annealing (recocido cuántico) para buscar soluciones a un problema. El recocido cuántico es fundamentalmente diferente de la computación clásica. Aprovecha la tendencia natural del mundo cuántico para encontrar estados de baja energía. El cálculo se realiza inicializando la unidad de procesamiento cuántico (QPU) en un estado básico de un problema conocido, aplicando el proceso de quantum annealing del sistema hacia el problema, se solucionará de modo que permanezca en un estado de baja energía durante todo el proceso. Al final del cálculo, cada qubit termina como 0 o 1 . Este estado final es la solución óptima o casi óptima al problema a resolver.

En la naturaleza, los sistemas físicos tienden a evolucionar hacia su estado de energía más baja: los objetos se deslizan cuesta abajo, las cosas calientes tienden a enfriarse y así sucesivamente. Este comportamiento también se aplica a los sistemas cuánticos. Para imaginar esto, piensa en un viajero que busca la mejor solución para encontrar el valle más bajo en el paisaje energético que representa el problema. Los algoritmos clásicos buscan el valle más bajo colocando al viajero en algún punto del paisaje y permitiendo al viajero moverse en base a variaciones locales. Si bien generalmente es más eficiente moverse cuesta abajo y evitar subir colinas que son demasiado altas, tales algoritmos clásicos son propensos a llevar al viajero a valles cercanos que pueden no ser el mínimo global. Normalmente se requieren numerosos ensayos, con muchos viajeros que comienzan sus viajes desde diferentes puntos. 
En contraste, el quantum annealing comienza con el viajero ocupando simultáneamente muchas coordenadas gracias al fenómeno cuántico de la superposición. La probabilidad de estar en cualquier coordenada dada evoluciona suavemente como el método progresa, con la probabilidad de aumentar alrededor de las coordenadas de los valles profundos. La Tunelización cuántica permite al viajero pasar por las colinas, en lugar de verse obligado a escalarlas, lo que reduce la posibilidad de quedarse atrapado en valles que no son el mínimo global. El entrelazamiento cuántico mejora aún más el resultado permitiendo al viajero descubrir correlaciones entre las coordenadas que conducen a valles profundos (DWAVE, 2018).

Pasar de un solo qbit a un QPU multi-qbit requiere que los qbits estén interconectados para intercambiar información. Los qbits se conectan mediante acopladores, que también son bucles superconductores. La interconexión de qubits y acopladores, junto con los circuitos de control para gestionar los campos magnéticos, crea un tejido de dispositivos cuánticos programables. Cuando la QPU llega a una solución de un problema, todos los qubits se asientan en sus estados finales y los valores que retienen se devuelven al usuario como una cadena de bits. El sistema D-Wave 2000Q tiene hasta 2048 qubits y 6016 acopladores. Para alcanzar esta escala, utiliza 128,000 uniones Josephson, lo que hace que este computador sea, con mucho, el circuito integrado superconductor más complejo jamás construido. (D-WAVE, 2018).

\subsection{APARIENCIA FÍSICA}

Un computador cuántico del modelo 2000Q tiene un tamaño de aproximadamente 10 'x 7' x 10 '(Largo x Ancho x Alto). Su espacio físico alberga un sofisticado sistema de refrigeración criogénica, blindaje y E / S para soportar una sola QPU del tamaño de una miniatura. La mayor parte del volumen del sistema se requiere para acomodar el sistema de refrigeración y para facilitar el acceso de servicio. Ya se había comentado que a los computadores cuánticos les afecta el calor y las perturbaciones externas, por eso, para que los efectos cuánticos desempeñen un papel en la computación, la QPU requiere un entorno extremo y aislado. El sistema de refrigeración y las capas de blindaje crean un ambiente interno de alto vacío con una temperatura cerca del cero absoluto que está aislado del exterior de los efectos de campos magnéticos, vibraciones y señales de RF de cualquier tipo. Gabinetes contiguos contienen los subsistemas de control, y los servidores front-end que proporcionan conectividad al sistema. (fig. 1) 
Fig. 1 Las entradas y salidas, el aislamiento y los materiales

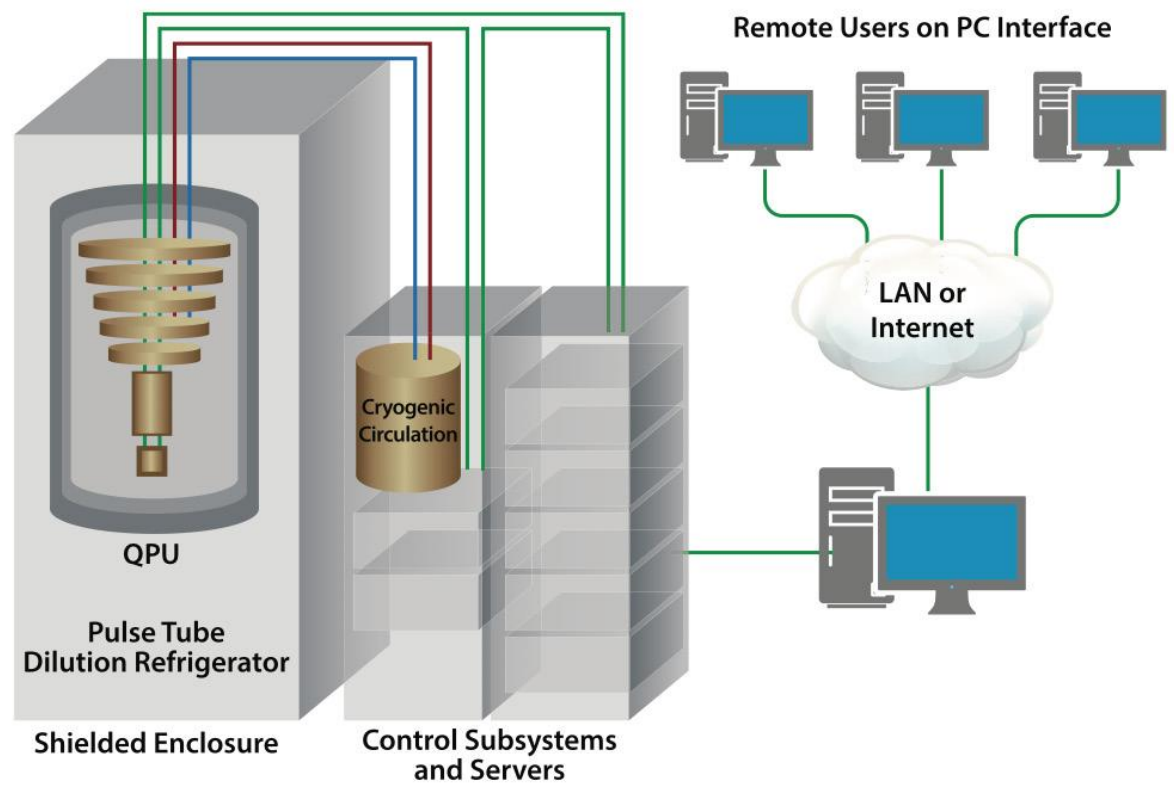

El entorno extremadamente aislado requerido para que el QPU funcione, coloca demandas inusuales en el diseño, de materiales y procesos de fabricación requeridos para los diversos subsistemas. El subsistema de E / S que pasa información a la QPU y viceversa filtra todo ruido no deseado y requiere una variedad de materiales normales y superconductores para proporcionar el rendimiento requerido. El subsistema de blindaje magnético proporciona un aislamiento del campo magnético muy alto, un entorno requerido para el QPU, usando alta capacidad de permeabilidad y materiales superconductores para lograr campos por debajo de 1 nanotesla. Esto es 50,000 veces menos que el campo magnético de la Tierra. El D-Wave QPU está construido a partir de una red de pequeños bucles de niobio metálico, cada uno de los cuales es un qbit. Por debajo de las temperaturas de $9.2{ }^{\circ} \mathrm{K}\left(-263.95{ }^{\circ} \mathrm{C}\right)$, el niobio se convierte en un superconductor y exhibe efectos mecánicos cuánticos.

\subsection{CONSUMO DE ENERGÍA}

Mientras que las supercomputadoras tradicionales generan cantidades masivas de calor y consumen enormes cantidades de energía, esta computadora cuántica consume menos de $25 \mathrm{~kW}$ de potencia, la mayoría de los cuales van hacia el funcionamiento de los servidores frontales y de enfriamiento. Este bajo consumo de energía se ha mantenido constante desde la introducción del primer sistema D-Wave a pesar del dramático aumento en rendimiento del sistema con cada generación sucesiva de productos. Los supercomputadores actuales consumen del orden de $2500 \mathrm{~kW}$ para modelos equiparables en capacidad de computo. 


\subsection{LAS APLICACIONES}

Las computadoras cuánticas son ideales para resolver muchos problemas difíciles de optimización, aprendizaje automático (machine learning), muestreo y seguridad cibernética. Con 2000 qubits y nuevas funciones de control, la computadora cuántica D-Wave 2000Q puede resolver problemas más grandes de lo que antes era posible, y con mejor rendimiento. Las comunidades crecientes de los desarrolladores están utilizando las capacidades únicas de estos computadores para resolver problemas difíciles en un conjunto diverso de áreas de aplicación que incluyen:

(a)En el campo de ciencias Computacionales: Detección de anomalías estadísticas. • Encontrar modelos

comprimidos. • Reconocer imágenes y patrones.

(b) Entrenamiento de redes neuronales. • Verificación y validación de software. • Clasificación de datos no

estructurados.

(c) Diagnóstico de fallas en el circuito.

(d) En el campo la seguridad y la planeación: • Detección de virus informáticos e intrusión de redes.

- Programación de recursos y rutas óptimas. Determinación del conjunto de miembros • Análisis de las

propiedades de gráficos. • Factorización de enteros.

(f) En el campo Financiero: Detectar inestabilidades del mercado. - Desarrollo de estrategias comerciales.

- Optimizando las trayectorias comerciales. - Optimización de precios de activos y cobertura. • Optimización

de carteras.

(g) En el campo de la Salud: Generando terapias dirigidas contra el cáncer. • Optimización de los tratamientos

de radioterapia. • Creación de modelos de proteínas.

\subsection{SOFTWARE Y PROGRAMACIÓN.}

El sistema D-Wave 2000Q proporciona una API de Internet estándar (basada en servicios RESTful), con bibliotecas disponibles para C / C ++, Python y MATLAB. Esta interfaz permite a los usuarios acceder al sistema ya sea como un recurso en la nube a través de una red, o integrado en sus entornos informáticos de alto rendimiento y centros de datos. El acceso también está disponible a través del servicio en la nube de D-Wave. Usando las herramientas de desarrollo de D-Wave y bibliotecas, los desarrolladores pueden 
crear algoritmos y aplicaciones dentro de sus entornos existentes utilizando herramientas estándar de la industria.

Cómputo Cuántico. El hardware está basado en la tecnología de los superconductores, y al igual que el software involucrado ambos están sustentados en la teoría de los qubits o bits cuánticos. Como sabemos, el cómputo convencional está basado en el sistema binario que representa dos estados 0's y 1's. Como vemos en Meglicki (2008), en mecánica cuántica tenemos el concepto de la esfera de Bloch (como se muestra en la figura 2), que representa espacios cuánticos de dos niveles; un ejemplo es el giro de los electrones, izquierdo o derecho. En un sistema de coordenadas cartesianas el punto $(0,0,1)$ representa el auto vector con autovalor positivo, mientras que el punto opuesto $(0,0,-1)$ equivale al auto vector con el autovalor negativo. En la terminología del cómputo cuántico, el punto de coordenadas equivalente a la esfera de Bloch representa un qubit, designando los estados como $\mid 0>$ y $\mid 1>$ respectivamente. Cada par de puntos diametralmente opuestos sobre la esfera de Bloch corresponde a dos estados ortonormales. Saltando todos los detalles llegamos a la matriz de Pauli, que es una matriz de 4 x 4, representando cada una de las posibilidades de cada octante $|0>0| 1>$. Esto equivale a que un estado que en binario era $0 \mathrm{o}$ 1, ahora tiene 16 posiciones con la posibilidad de un 0 o un 1 . A final de cuentas, esto llevado a la práctica representó enviar y procesar en el mismo espacio muchos más datos.

Figura 2. Dígito Binario o Bit, y el bit cuántico o qubit.

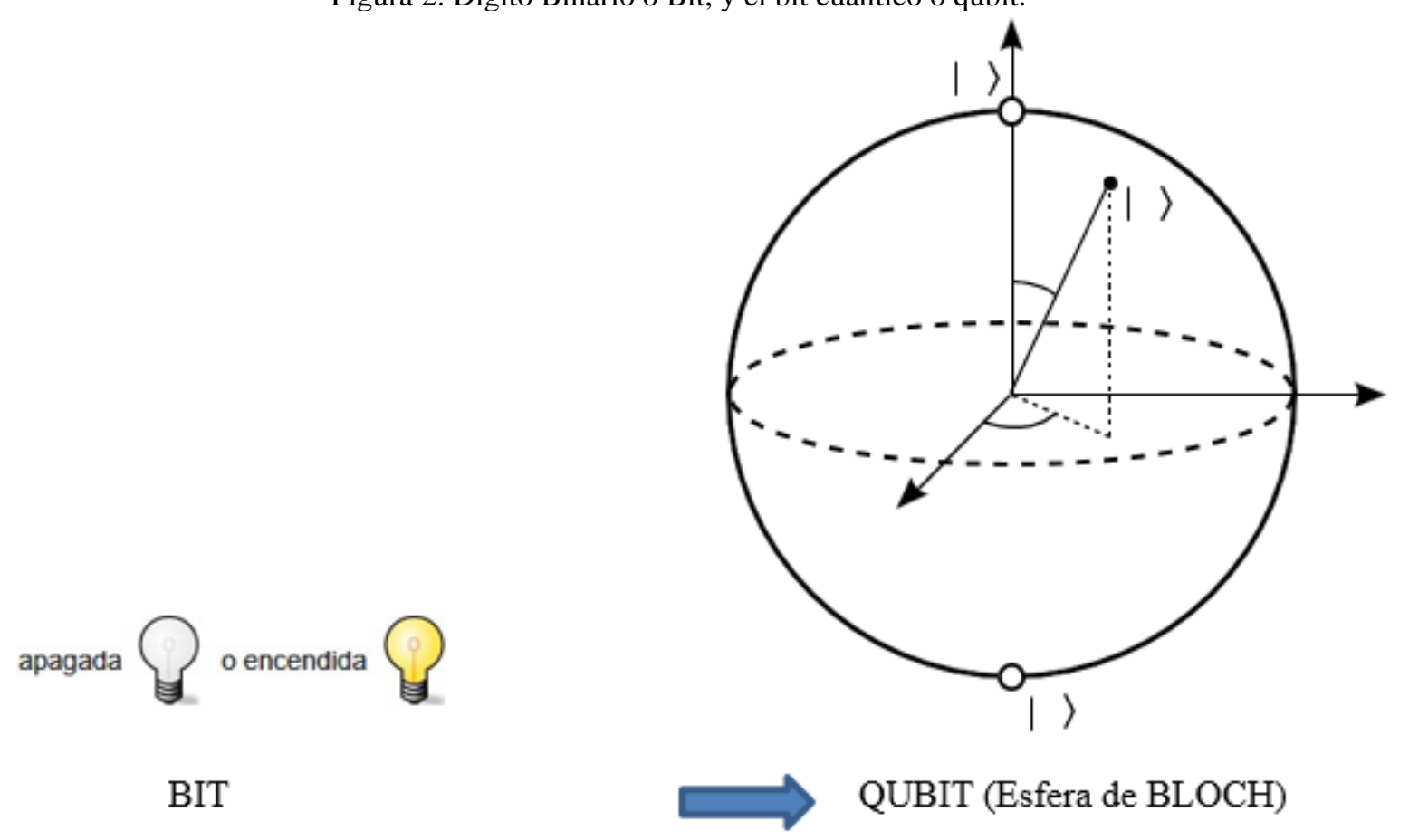


La diferencia en capacidad de cómputo resultó espectacular, aumentando la velocidad de procesamiento en un promedio de mil veces más a un computador cuántico comparado con uno convencional. Para dar una idea, una computadora cuántica de 30 qubits, equivale a un microprocesador convencional de 10 teraflops (10 millones de millones de operaciones de punto flotante por segundo), comparado con las computadoras convencionales que operan en el orden de gigaflops (miles de millones de millones de operaciones por segundo) (Meglicki, 2008).

El aspecto de la velocidad de procesamiento es mucho más complejo, como vemos en D-WAVE (2018b) la codificación del qubit como 0s, 1s, o ambos simultáneamente, en una superposición de estados. Dicha superposición de estados, además de los efectos de entramado y túnel cuántico, hacen posible que las computadoras cuánticas consideren y manipulen muchas combinaciones de bits simultáneamente. Contrario a lo que se podría esperar dado el gran incremento de velocidad de proceso, el consumo de energía es aproximadamente mil veces menor, por ejemplo, si el sistema D-Wave 2000Q consume $25 \mathrm{Kw}$ en un período determinado de tiempo, un sistema tradicional de supercomputadoras equivalente consumiría 2,500 Kw (D-WAVE, 2018b)

El Lenguaje de Programación Q\#. De acuerdo a Microsoft (2017), un modelo natural para la computación cuántica es tratar a las computadoras cuánticas como un co-procesador, similar a los que se usan para las unidades de procesamiento de gráficos (GPUs, por sus siglas en inglés), matrices de compuertas programables (FPGA, por sus siglas en inglés) y otros procesadores adjuntos o adicionales al microprocesador principal. El control lógico primario ejecuta código clásico sobre una computadora huésped (Host). Cuando es apropiado y necesario, el programa huésped puede invocar a un sub programa que se ejecuta en el procesador adjunto. Cuando el sub programa termina, el programa huésped obtiene acceso a los resultados del sub programa. Q\# (Q-sharp) es un lenguaje de programación de dominio específico usado para expresar algoritmos cuánticos. Este es usado para escribir sub programas que se ejecuten en un procesador cuántico adjunto, bajo el control de una computadora y programa huésped clásicos. Q\# proporciona un pequeño conjunto de tipos primitivos, a través de dos caminos, arreglos y registros, para crear nuevos tipos estructurados. Soporta un modelo procedural básico para escribir programas, con ciclos y sentencias condicionales. El nivel principal de constructores en Q\# son los tipos de datos definidos por el usuario, operaciones, y funciones (Microsoft, 2017).

EI Caso Google. El conocido buscador para la web, el Google Search, como podemos ver en la publicación de su creación Brin y Page (1997), nace como un motor de búsqueda a gran escala. El prototipo tenía la capacidad para procesar 24 millones de páginas. Cabe destacar que ya consideraba el manejo de grandes archivos (páginas), a través de su componente BigFiles, que podía segmentarlos en múltiples archivos para su almacenamiento (Brin y Page, 1997). Scarpinelli (2013) nos muestra una estadística sorprendente: Google procesa 100,000 millones de búsquedas por mes, en 159 idiomas. Cada 
solicitud viaja un promedio de 2,400 kilómetros en $1 / 4$ de segundo, tiempo que tarda en surgir la respuesta, de entre algo así como 60 trillones de páginas web. En opinión de sus fundadores Larry Page y Sergei Brin, Google es la puerta de acceso a un repositorio casi infinito de información, que más allá de su umbral, está organizada y accesible (Scarpinelli, 2013).

Las Computadoras Cuánticas. De acuerdo a D-WAVE (2018), la empresa D-Wave que se funda en 1999 con el objetivo de llevar a la práctica el cómputo cuántico, logra su propósito tras cinco años de investigaciones, lanzando al mercado su primera computadora cuántica en 2010. Iniciando con un modelo de 512 qubits, llega a su modelo de 2000 qubits en 2017. Estos modelos que han incrementado en miles de veces la capacidad de cómputo oscilan en el orden de los 10 millones de \$USD (D-WAVE, 2018). En este sentido, esta capacidad de cómputo de alto rendimiento resulta muy onerosa aún para las grandes empresas. En este tenor, como vemos en IBM (2017) la International Business Machinery (IBM) ha dirigido su estrategia totalmente diferente a D-Wave; su objetivo es la construcción de sistemas IBM Q, del orden de 50 qubits, capacidad muy por debajo de la D-Wave, pero con dos grandes ventajas: (a) Económicamente más accesible y (b) Disponible a través de la nube. En esta estrategia conocida como IBM Quantum Experience, desde su lanzamiento a finales de 2016, alrededor de 40 mil usuarios han realizado 275 mil experimentos. Lo anterior ha sido gestionado por el IBM Research Frontiers Institute, consorcio que ha agrupado al MIT, el Institute for Quantum Computing at the University of Waterloo, la European Physical Society, incluyendo empresas como Samsung, JSR, Honda, Canon, Hitachi Metals y Nagase (IBM, 2017). Estos aspectos nos dan idea del crecimiento de los datos y su naturaleza (big data), que a la par con su crecimiento exponencial, demanda enormes recursos computacionales.

El almacenamiento y clasificación para su posterior recuperación, además de la diversidad en el tipo de datos, está llevando al límite a las computadoras actuales. Además, como ya se mencionó, existen problemas o algoritmos cuánticos que costaría mucho trabajo programarlos en las computadoras convencionales, inclusive algunos no se pueden programar, o no son viables debido al tiempo de respuesta. Como vemos en Hutterer (2016), "Einstein no estaba totalmente convencido, pero la mecánica cuántica hoy es aceptada como el camino del mundo". Las partículas que interactúan entre sí entran en una relación extraña. Esta relación, conocida como entramado, se conserva siempre que las dos partículas permanezcan protegidas del resto del ambiente, para que su enredo no se separe. Por ejemplo, si dos electrones fueran enredados de tal manera que necesariamente deben girar en la misma dirección, pero inicialmente están en una superposición de ambas direcciones posibles, el instante en que uno de ellos asume una orientación de giro firme (debido a una medida, tal vez), el otro asume la misma orientación también. Con una marca burlona de Einstein como "acción espeluznante a distancia", este fenómeno se mantiene incluso si los dos electrones se han separado miles de años luz. La superposición, la decoherencia y el entramado son, sin duda, un gran desafío, pero eso no los hace menos reales. 
Si estos extraños principios de la ciencia pueden ser aprovechados de alguna manera, entonces podría ser posible hacer retroceder algunas cortinas. Cableando la rareza, una computadora clásica usa bits como unidades de información. El término "bit" proviene de "dígito binario", que ilustra su naturaleza de dos estados: siempre debe esta en uno de los dos estados, que se denota con un 1 o un 0 . Los dos estados pueden ser casi cualquier conjunto binario

- abierto y cerrado, encendido y apagado, arriba y abajo, positivo y negativo - y el estado del bit describe el estado de alguna parte del dispositivo físico.

Una computadora cuántica, por otro lado, se basa en bits cuánticos o "qubits" como unidades de información. En la figura 3 se observa la D-Wave 2X, que es una gran caja negra con una huella de 150 pies cuadrados (incluido el gabinete de controles contiguo). Esencialmente es un congelador empotrado que está altamente protegido contra interferencias externas, creando un entorno especializado para el primer procesador cuántico comercial del mundo. El entorno alrededor del chip tiene un campo magnético 50,000 veces más débil que el campo magnético ambiental de la Tierra, una presión de 10 mil millones de veces más baja que la presión atmosférica de la Tierra y una temperatura cientos de veces más fría que el espacio interestelar.
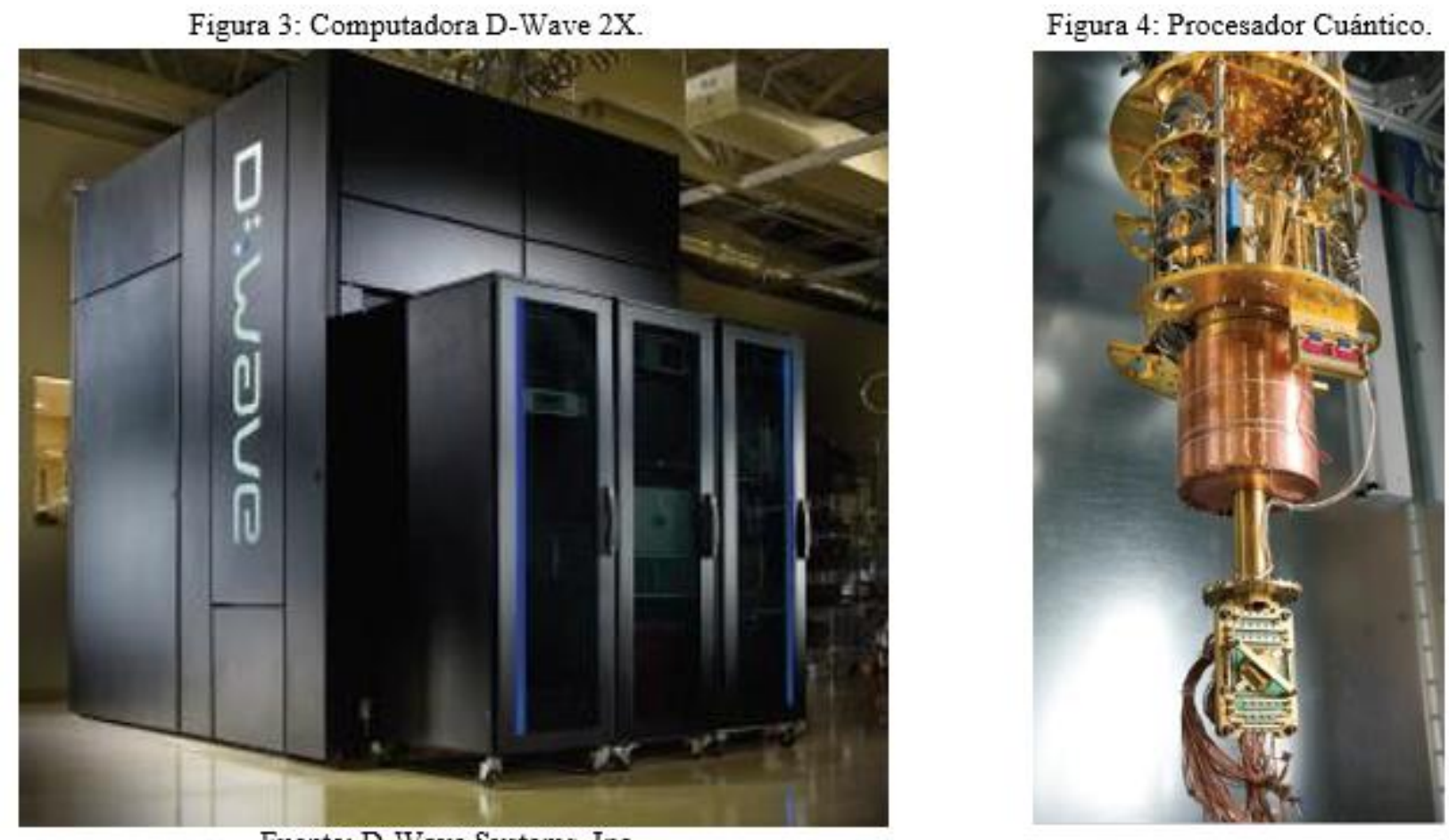

Fuente: D-Wave Systems, Inc

La figura 4 muestra un conjunto de 16 capas de blindaje y filtros (que se eliminaron para mostrar el dispositivo en el interior) que sirven para reducir drásticamente la temperatura e interferencia con el procesador cuántico (visible en la parte inferior detrás de una barra de oro diagonal) (Hutterer, 2016).

Qubit. Como vemos en D-WAVE (2018c), la velocidad de procesamiento de las computadoras 
cuánticas está sustentada por las leyes de la mecánica cuántica. El almacenamiento de la información usa representaciones de bits (0's y 1's) como lo hacen las computadoras convencionales, pero ahora utiliza bits cuánticos o qubits, que pueden codificar ceros y unos simultáneamente. Este estado de superposición además de los efectos cuánticos de entanglement (entramado) y tunneling (tunelización), permiten manipular muchas combinaciones de bits simultáneamente. Las computadoras cuánticas se apoyan en dinámicas cuánticas para acelerar y ofrecer nuevos métodos para optimización discreta y problemas de machine learning. Utilizan un proceso denominado quantum annealing (templado cuántico) para buscar soluciones a problemas de búsqueda. El cómputo es realizado inicializando la unidad de procesamiento cuántico (QPU por sus siglas en inglés) dentro de un estado basal de un problema conocido, y templado o normalizado hacia la solución del problema, permaneciendo siempre en un estado de baja energía a través del proceso.

Al final del cómputo, cada qubit termina siendo un cero o un 1; este estado final es la solución óptima al problema resuelto. Ir de un qubit simple a un multi - qubit QPU, requiere que los qubits sean interconectados para intercambiar información. Los qubits son interconectados vía couplers (acopladores), los cuales son como ciclos o lazos superconductores. La interconexión de qubits y acopladores, junto con la circuitería para administrar los campos magnéticos, crea una fábrica integrada de dispositivos cuánticos programables. Cuando el QPU llega a la solución de un problema, todos los qubits se establecen dentro de su estado final y sus valores son retornados al usuario como una cadena de bits. Para darnos mayor idea de la complejidad, mencionemos el modelo D-Wave 2000Q, que cuenta con 2048 qubits y 6016 acopladores. Para alcanzar esta estructura, utiliza 128,000 juntas de Josephson, haciendo de este QPU un enorme y complejo circuito integrado superconductor (D-WAVE, 2018c).

Algoritmos Cuánticos. De acuerdo con Goddard, Mniszewski, Neukart, Pakin, \& Reinhardt (2017), Scott Pakin del laboratorio nacional de los álamos describe los sistemas de cómputo cuántico en su implementación de dos modelos computacionales: el puente o modelo circuito, y el quantum annealing (QA, templado o normalizado cuántico). Un procesador basado en QA es un dispositivo de propósito especial, que nativamente resuelve problemas de quadratic unconstrained binary optimization (QUBO, optimización binaria cuadrática no restringida). El objetivo de un QUBO es encontrar la xi $\in$ $\{0,1\}$ que minimice $x^{\mathrm{T}} \mathrm{Qx}$ para una matriz triangular superior $\mathrm{Q}$ con $\mathrm{Qi}, \mathrm{j} \in \mathrm{R}$. El QUBO es equivalente al unconstrained binary quadratic programming (UBQP, programación cuadrática binaria no restringida).

Uno puede pensar del templado o normalizado cuántico como una implementación de hardware que simula los efectos cuánticos de superposición, entramado y tunelado, para reducir lo esperado a un mínimo local. En ambas normalizaciones cuánticas simuladas, encontrando la xi que verdaderamente 
minimice (1) no está garantizado. Sin embargo, la meta es normalmente encontrar una buena solución rápidamente. Una computadora D-Wave puede proponer una solución a (1) en solo 5 microsegundos, para N > 2000, donde el espacio de búsqueda es más grande que 2000 (Goddard, Mniszewski, Neukart, Pakin, \& Reinhardt, 2017).

El Algoritmo de Shor: Como describe Darwish (s/f), los métodos modernos para la descomposición de números grandes en sus factores primos son ineficientes; este hecho es el que sustenta la seguridad en los mecanismos de encriptación de llave pública y privada basados en números primos. Encontró que por ejemplo, para números con 130 dígitos, aplicar el método de Shor en una computadora cuántica no aporta ventajas sobre uno clásico, empleando aproximadamente 7 horas a una tasa de conmutación de un MHz; pero con 260 dígitos, el computador cuántico tarda 8 veces más. Pero, para el computador clásico esto es intratable, es decir, no obtiene una solución en un tiempo razonable. Lo anterior es debido a que el método de Shor explota la potencia de las características de la mecánica cuántica de entrelazamiento, interferencia y paralelismo cuántico, aplicándolas a la transformada cuántica de Fourier que es la base del método.

El algoritmo de búsqueda de Grover: Desde el punto de vista de la mecánica cuántica, podemos utilizar el algoritmo presentado por Grover en 1997. Partiendo de una lista desordenada $\{\mathrm{xi}\} \mathrm{Ni}=1$, donde queremos localizar al elemento $\mathrm{xj}=\mathrm{t}$, tenemos que un algoritmo clásico, que recorre la lista de $\mathrm{N}$ elementos, requiere en promedio realizar N/2 comparaciones, mientras que el método de Grover requerirá solamente $\sqrt{ } \mathrm{N}$. El método es más eficiente cuanto más grande es la lista, logrado esto al aprovechar la superposición, a través de la transformada cuántica de Fourier (Darwish, s/f).

El Lenguaje Q Sharp (Q\#). Los lenguajes nativos de la D-Wave 2000Q son el C / C++, Matlab y Python. Se espera que pronto haya una versión de Visual Studio para la programación cuántica. De acuerdo con Mykhailova, Paz, \& Kinney (2017), una tecnología emergente de los últimos años es el cómputo cuántico, cambiando los grandes paradigmas para los desarrolladores de software. Las computadoras cuánticas fueron propuestas en 1980 por Richard Feyman y Yuri Manin, y la intuición detrás del cómputo cuántico surgió como un gran reto de la física: un notable progreso científico ante la imposibilidad de modelar esos sistemas cuánticos. La mecánica cuántica se desarrolló entre 1900 y 1925 y sigue siendo la piedra angular de la química, la física de materia condensada, y tecnologías que van desde microprocesadores hasta iluminación LED.

A pesar de esos éxitos, algunos sistemas parecen estar más allá de la capacidad humana para su modelado cuántico. Esto es debido a que la simulación de partículas interactuantes requiere más potencia informática que cualquier ordenador convencional. Hay muchas formas de entender por qué la mecánica cuántica es difícil de simular. Lo más sencillo es ver que la teoría cuántica puede interpretarse diciendo 
que la materia a nivel cuántico es al mismo tiempo una gran cantidad de diferentes configuraciones posibles o estados. A diferencia de la teoría clásica de la probabilidad, esas muchas configuraciones del estado cuántico que potencialmente pueden observarse pueden interferir entre sí como olas en una alberca; esta interferencia evita el uso de muestreo estadístico para obtener las configuraciones de estado cuántico.

Por el contrario, tenemos que rastrear cada posible configuración del sistema cuántico si queremos entender la evolución cuántica. Por ejemplo, considerando un sistema de electrones donde los electrones pueden estar en cualquiera de 40 posiciones, los electrones, por tanto, pueden estar en cualquiera de 240 configuraciones (ya que cada posición puede tener o no tener un electrón). Para almacenar el estado cuántico de los electrones en una memoria de ordenador convencional requeriría de 130 GB de memoria. Esto es importante, pero al alcance de algunos equipos. Si permitimos que las partículas existieran en cualquiera de 41 posiciones, habría dos veces muchas configuraciones en 241, que a su vez requerirá más de 260 GB de memoria para almacenar el estado cuántico. Este juego de aumentar el número de posiciones no se puede reproducir indefinidamente si queremos almacenar el estado convencionalmente, ya que rápidamente excedemos la capacidad de memoria de las máquinas convencionales más poderosas disponibles.

El núcleo fundamental de la computación cuántica es para almacenar información en los estados cuánticos de la materia y utilizar las operaciones de la puerta cuántica para calcular en esa información, para aprovechar y aprender de la interferencia cuántica. Un ejemplo temprano de la interferencia de programación para resolver un problema se cree que en nuestros ordenadores convencionales fue realizado por Peter Shor en 1994 en un problema conocido como factoring (factorizado). La solución de factoring trae consigo la capacidad de romper muchos de nuestros criptosistemas de clave pública subyacente a la seguridad del comercio electrónico hoy en día, como RSA y la criptografía de curva elíptica. Desde entonces, se han desarrollado algoritmos cuánticos rápidos y eficientes para muchas de nuestras tareas clásicas: simulación de sistemas físicos en química, física y ciencia de los materiales, la búsqueda desordenada de una base de datos, resolución de sistemas de ecuaciones lineales y el aprendizaje de máquina(machine learning) (Mykhailova, Paz, \& Kinney, 2017).

\section{PRUEBAS Y RESULTADOS}

El diseño de un programa de procesamiento cuántico para aprovechar la interferencia puede sonar como un desafío de enormes proporciones, y en realidad lo es, pues existen muchas técnicas y herramientas, incluyendo el Kit de desarrollo de Microsoft cuántico, que se ha introducido para hacer programación cuántica y desarrollo de algoritmos más accesible. Hay un puñado de estrategias básicas que pueden utilizarse para manipular la interferencia cuántica de una forma útil para la informática, 
mientras que al mismo tiempo nos da la solución para luego perderse en una maraña de posibilidades cuánticas. Programación cuántica es un arte distinto de la programación clásica que requiere diferentes herramientas para comprender y expresar el pensamiento algorítmico cuántico. De hecho, sin necesidad de herramientas generales para ayudar a un desarrollador de quantum en abordar el arte de la programación cuántica, el desarrollo algorítmico del quantum no es tan fácil.

\section{CONCLUSIONES}

Como podemos ver la CQ es una realidad. Con casi tres décadas de desarrollo e investigación se ha logrado construir máquinas que al día de hoy se pueden comercializar. Los precios de estos productos siguen siendo mucho muy elevados para el consumo masivo por parte de los usuarios que hoy poseen un computador clásico en su hogar. Pero las grandes corporaciones ven en la CQ posibles soluciones a problemas de cómputo que por ahora requieren de supercomputadoras con un consumo de energía muy elevado. Además, la posibilidad de resolver problemas que requieren una cantidad inmensa de cómputo y que por ahora es muy difícil resolverlos o casi imposible, hace muy atractivo el desarrollo de esta tecnología ya sea por parte de los fabricantes como de los centros de investigación, las corporaciones financieras y aquellas compañías que por la naturaleza de los servicios que prestan es necesario tener equipos con muy altas prestaciones de velocidad y manejo masivo de datos.

El Kit de desarrollo cuántico de Microsoft va dirigido a empoderar a una comunidad en crecimiento con herramientas para desbloquear la revolución cuántica para sus tareas, problemas y soluciones. El lenguaje de alto nivel, Q \#, fue diseñado para enfrentar los desafíos de procesamiento de información cuántica, está integrado en una pila de software que permite a un algoritmo cuántico compilar hasta las operaciones primitivas de un ordenador cuántico. Así como sucedió hace ya más de 25 años cuando hubo una migración general de la programación estructurada a la programación orientada a objetos, es tiempo de ir viendo la migración a la computación cuántica. 


\section{REFERENCIAS}

Da Costa, A., Goncalves, J., Neta, H., Alves, D., Franceschi, E., Dariva, C., . . Leite, M. (2020). Machine learning applied as an in-situ monitoring technique for the water content in oil recovered by means of NIR spectroscopy. South Florida Journal of Development., 2(1), 405-416.

Darwish, N. (s/f). Computación Cuántica. Facultad de Ciencias Exactas, Ingeniería y Agrimensura. Obtenido de https://www.fceia.unr.edu.ar/ diazcaro/QC/Tutorials/Computacion\%20Cuantica.pdf

D-WAVE. (25 de Enero de 2018). D-Wave Systems Inc. Obtenido de https://www.dwavesys.com/ourcompany/meet-d-wave

D-WAVE. (2018b). D-Wave Systems Inc. Obtenido de https://Wave\%202000Q\%20Tech\%20Collateral_0117F.pdf

D-WAVE. (2018c). D-Wave Systems Inc. Obtenido de https://www.dwavesys.com/sites/default/files/DWave\%202000Q\%20Tech\%20Collateral_0718web.pdf

Goddard, P., Mniszewski, S., Neukart, F., Pakin, S., \& Reinhardt, S. (01 de Diciembre de 2017). How Will Early Quiantum Computing Benfit Computational Methods? Obtenido de https://www.dwavesys.com/sites/default/files/SIAM_December_2017_How_Will_Early_Quantum_Co mputing_Benefit_Computation al_Methods.pdf

Hutterer, E. (2016). Not Magic Quantum. Recuperado el 07 de Diciembre de 2018, de https://www.dwavesys.com/media/b4penug4/1663_july-2016-not-magic-quantum.pdf

IBM. (07 de marzo de 2017). IBM Construye Primeras Computadoras Cuánticas Universales para Aplicaciones Empresariales y Científicas.Obtenido de https://www03.ibm.com/press/mx/es/pressrelease/51778.wss

Meglicki, Z. (2008). Quantum Computing Without Magic: Devices. Obtenido de http://web.a.ebscohost.com.ezproxylocal.library.nova.edu/ehost/ebookviewer/ebook/bmxlYmtfXzIzNzc 2N19fQU41?sid=343481cd-6e49-4279-bc3b-9ecaf31344e6@ sessionmgr4009\&vid=3\&format=EB

Microsoft. (12 de noviembre de 2017). The Q\# Programming Language. Obtenido de https://docs.microsoft.com/en-us/quantum/quantum-qr- intro?view=qsharp-preview

Microsoft. (2018). Get started with quantum development. Obtenido de https://www.microsoft.com/enus/quantum/development-kit

Moret, V. (2005). Principios Fundamentales de Computacion Cuantica. Obtenido de https://enginyeriainformatica.cat/wp-content/uploads/2016/05/PRINCIPIOS-FUNDAMENTALES-DECOMPUTACIÓN-CUÁNTICA.pdf

Mykhailova, M., Paz, A., \& Kinney, A. (10 de Septiembre de 2017). Welcome to the Microsoft Quantum Development Kit Preview. Obtenido de https://docs.microsoft.com/en-us/quantum/index?view=qsharppreview

Scarpinelli, L. (06 de Octubre de 2013). La Nación. Obtenido de Google: 15 años de la empresa que cambió el mundo: http://www.lanacion.com.ar/1626139-google-15-anos-de-la-empresa-que-cambio-elmundo

Soler-Zapata, M., Franco-Escudero, E., \& Arceo-Reyes, R. (2021). Un algoritmo recursivo en paralelo usando la ecuación radial de onda. South Florida Journal of Development, Miami, 2(5), 7865-7871. 


\section{NOTAS BIOGRÁFICAS}

El Dr. José Ruiz Ayala es profesor investigador en el Tecnológico Nacional de México / Tecnológico de la Laguna, en Torreón, México. Tiene Maestría en Sistemas Computacionales Administrativos, del Instituto Tecnológico y de Estudios Superiores de Monterrey y Doctorado en Liderazgo Instruccional y el Currículum, de la Universidad Nova Southeastern, de Miami, FL.

El Dr. Luis Héctor García Muñoz es profesor investigador en el Tecnológico Nacional de México / Tecnológico de la Laguna en Torreón, Coah. México. Es Doctor en Informática por la Universidad Politécnica de Valencia, España.

El MC. Antonio de Santiago Barragán es profesor investigador en el Tecnológico Nacional de México / Tecnológico de la Laguna en Torreón, Coah. México, Tiene Maestría en Sistemas Computacionales por el Tecnológico Nacional de México / Tecnológico de la Laguna y es jefe de la carrera de Ingeniería en Sistemas Computacionales.

EL Ing. Alberto Medina Rodríguez es estudiante de la maestría en sistemas computacionales en el Tecnológico Nacional de México / Tecnológico de la Laguna en Torreón, Coah. México. 